\title{
A ASSOCIATION OF FEMALE ATHLETE TRIAD TO OTHER PSYCHO-PHYSIOLOGICAL PROBLEMS
}

Tanugatri Majumder

MSc. Sports Science(2019-2021)

Ramakrishna Mission Vivekananda Educational and Research Institute (RKMVERI), Belur Math, India.

INTRODUCTION

The Female Athlete Triad is a comr

low energy availability with or without disordered eating

- menstrual dysfunction and

* low bone mineral density (BMD) leading to Osteopeniz

and susceptible to stress fractures.

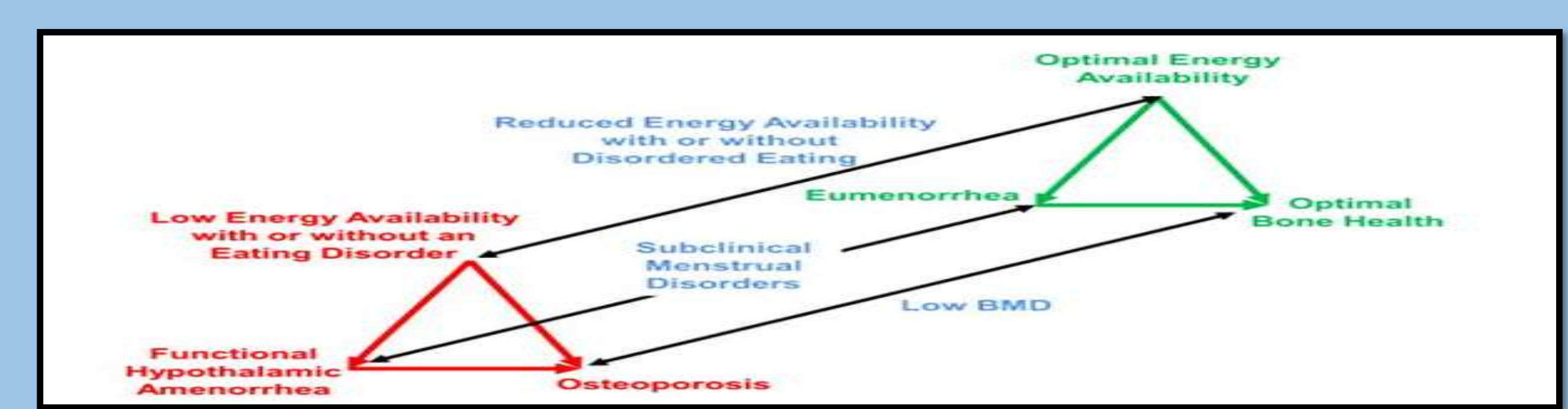

Various endrocrinological, physiological and psychological problems associated with the Triad can lead to significant health disorders with time. To prevent and trea Sportspersons at risk or affected with the Triad, it is important to follow guidelines for early detection, prevention and treatment. Prevention tactics should focus on health
education and counseling, regular screenings and medical support for athletes to increase health knowledge that could prevent the development of this syndrome. Female athletes should be able to keep track of their menstrual phases and address their physiological and behavioural changes (or patterns) associated with their rhythm. Introducin elevant nutritional, motivational and training changes accordingly can enhance the Athlete's overall performance.

\section{LOW ENERGY AVAILABILITY and EATING DISORDERS}

Disordered Eating involves disturbance in an athlete's diet or in the way one experiences their body, excessive or rigid exercise routine, obsessive calorie counting, anxiety about certain food groups and choosing a rigid approach to eating.

Extreme weight loss methods, severe calorie and water restrictions combined with vigorous exercise, can lead to disturbed eating behavior, anxiety, depression, and clinical Eating disorders(EDs).

The continuous restrictive eating and malnutrition results in pervasive disturbances of most organ systems including cardiovascular and gastrointestinal complications, endocrine disorders (i.e. osteopenia and amenorrhea) and other metabolic alterations.

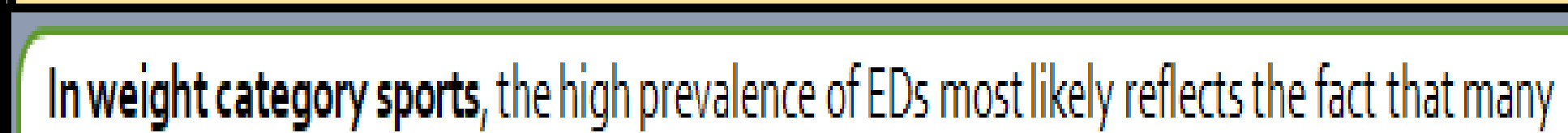

\section{athletes want to havea low body
classbelow their ordinaryweight.}

Athletescompetinginsports

that emphasize a lean body shape or a low bodyweight showed a significantlyhigher

\section{symptoms and dinically}

\section{Clagnosedebs than on}

gymnastics, ballet, figure skating, lightweight rowing,

\section{and running are at risk duet}

pressuretomaintain a low body weight along with poor guidance
aboutnutrition and weightl loss.
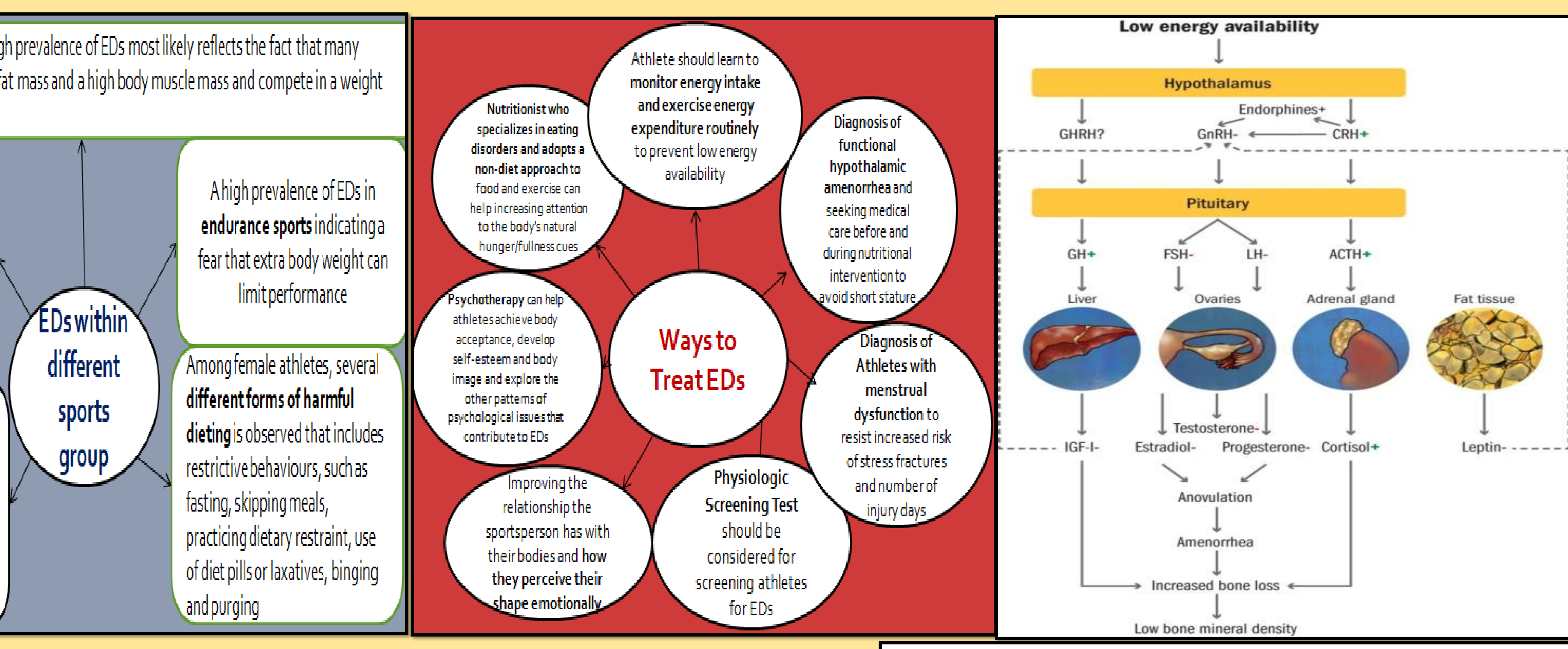

- Athletes unknowingly fail to attain their energy requirements or lack of nutritional knowledge neces to promote food intake as compensation $\mathrm{f}$
causes Low Energy Availability (LEA). * Wide spectrum of disordered eating that ranges from excessive dieting to clinical disorders such as
Anorexia nervosa, Bulimia nervosa, eating disorder otherwise not specified (EDNOS) and other prevalent psychiatric disorders is often observed

* Deficiency in macronutrients can affect body's ability to build bone, maintain muscle mass and repai damaged tissue. Thus, it is important to maintain a recommended diet plan with a proper ratio of macronutrients, micronutrients and roughage.

\section{MENSTRUAL DYSFUNCTION in the Female athletes}

Menstrual dysfunction in the female athletes includes a wide spectrum of disorders:

- Amenorrhea (absence of menses 3 months, delay or early onset of menarche), anov
by energy deficiency and stress is Functional Hypothalamic Amenorrhea (FHA).

- In amenorrheic athletes, decrease in fat mass and Leptin deficiency also contributes to the loss of menses.

- The disruption of lutein
hypothalamic origin.

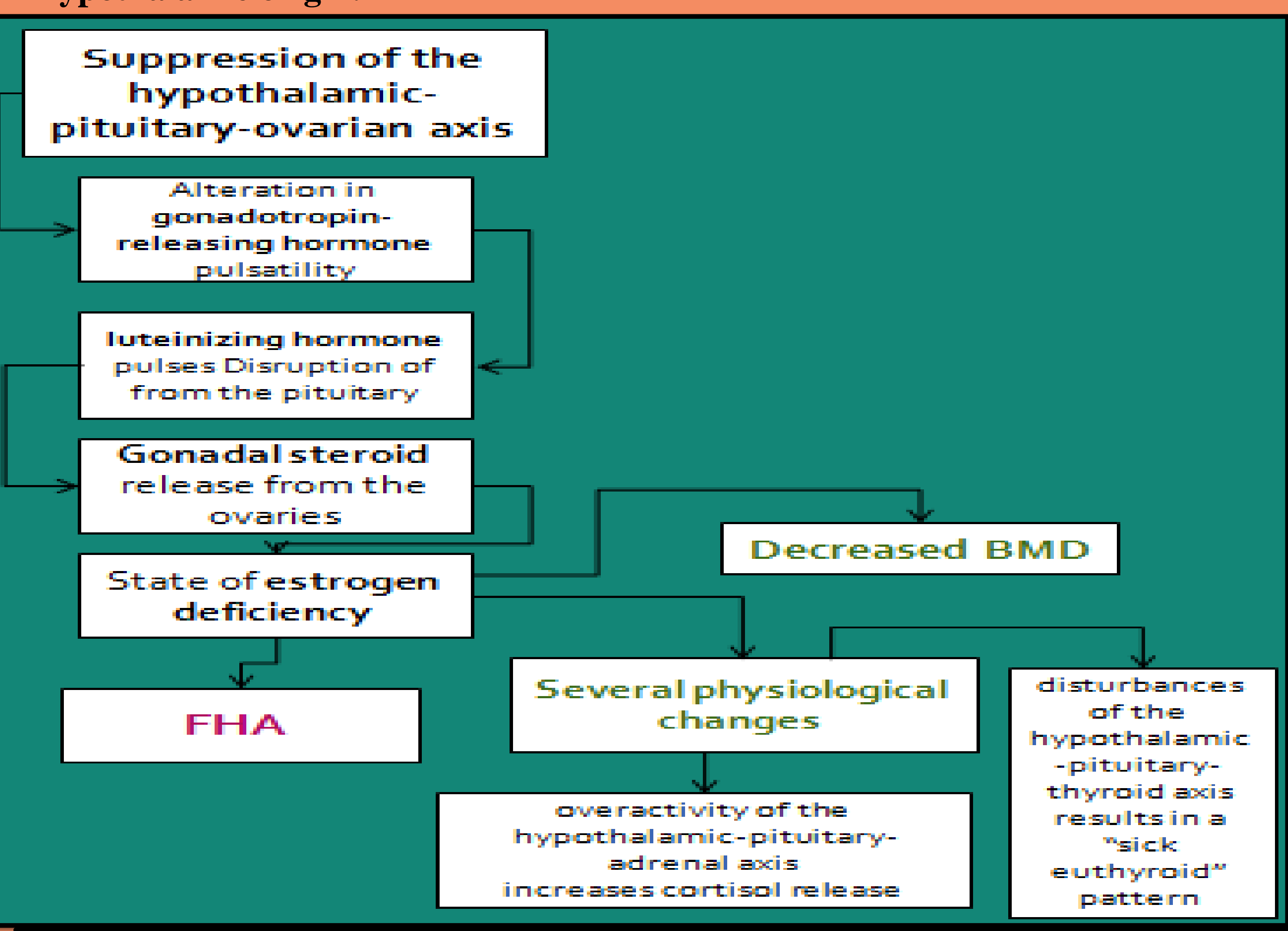

ALTERED ENDOCRINE FACTORS That affect sport performance:

- Nonsteroidal anti-inflammatory drugs (NSAIDs) taken for dysmenorrhea, affects airway resistance through prostaglandin inhibition and cause menstrual-linked asthma or perimenstrual asthma.

Therapeutic usage of hormones for menstrual manipulation and withdrawal bleeding has un

An increasing number of athletes use Oral Contraceptive Pills (OCP) for cycle control.

Hypoestrogenism resulting from a suppression of hypothalamic pulsatile release of gonadotropin-

releasing hormone $(\mathrm{GnRH})$ and decline in the concentration of Luteinizing Hormone (LH) and

Follicle Stimulating Hormone(FSH).

Abnormal Oestrogen (E) and progesterone (P) rhythms alters energy substrate metabolism, thermoregulation, body water and electr

and makes the athlete prone to injury.

Hormonal changes due to FHA includes:

Increase in levels of Neuropeptide Y, Ghrelin, Corticotropin Releasing Hormone, Cortisol and highe pulsatile amplitude of Allopregnanolone.
Menstrual dysfunctions may override the positive effects of exercise on BONE HEALTH in young adults:

Bone strength, as a triad component, occurs along a spectrum that spans from low bone mass and
condition.

- The greatest increase of bone mass happens during puberty (11- 14 years). Young healthy women achieve $92 \%$ of their total body bone mineral content (peak bone mass) by 18 years and $99 \%$ by age 26 years.

- Optimum consumption of Calcium, vitamin D, vitamin $\mathrm{K}$ and fish oil is important for bone health (1300 mg of calcium and $600 \mathrm{IU}$ of vitamin D daily). Bisphosphonates are not recommended for bone density as it can result in a potential teratogenic effect. which results in low BMD, stress fractures and osteoporosis. The process of Bone turnover (of bone formation and matrix development by osteoblasts and bone breakdown by osteoclasts) is interrupted, bone becomes weakened and prone to injury.

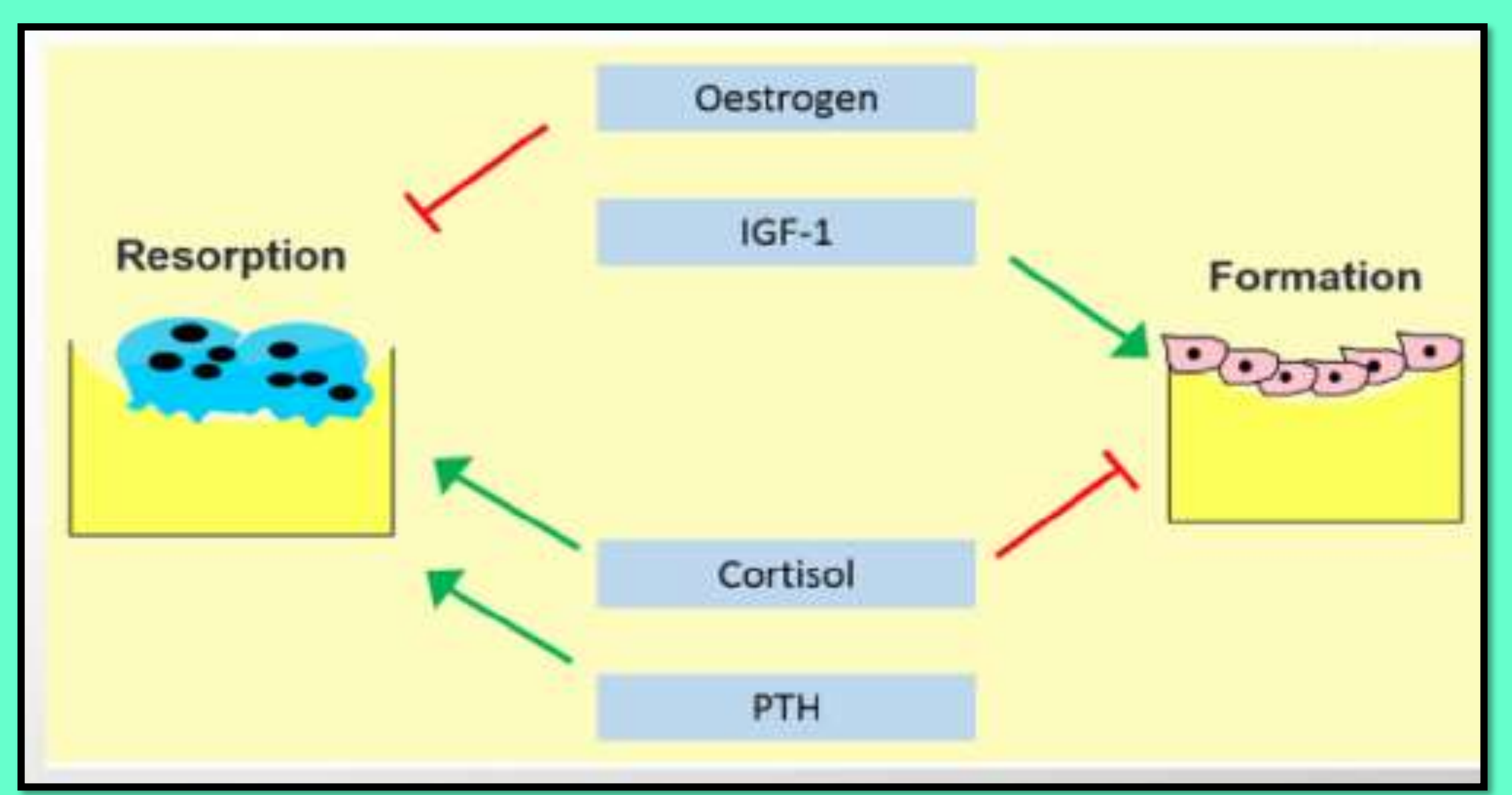

Weightbearing exercise affects bone accrual and architecture. Amenorrheic athletes have overall lower BMD, $10 \%$ to $20 \%$ less lumbar spine BMD and 2 to 4 times greater risk for stress fracture than their eumenorrheic counterparts. Oligomenorrhea and amenorrhea (hypoestrogenic states) cause disruption of bone

Resumption of menses cannot solve BMD issues immediately but gradual rebuilding of bone occurs to decrease the risk of future osteoporosis and injury. Depending on the age of the athlete, duration of the triad, and time to recover, BMD may improve but not reach the normal levels.

Hormonal rhythm regulating Bone health in Female Athetes

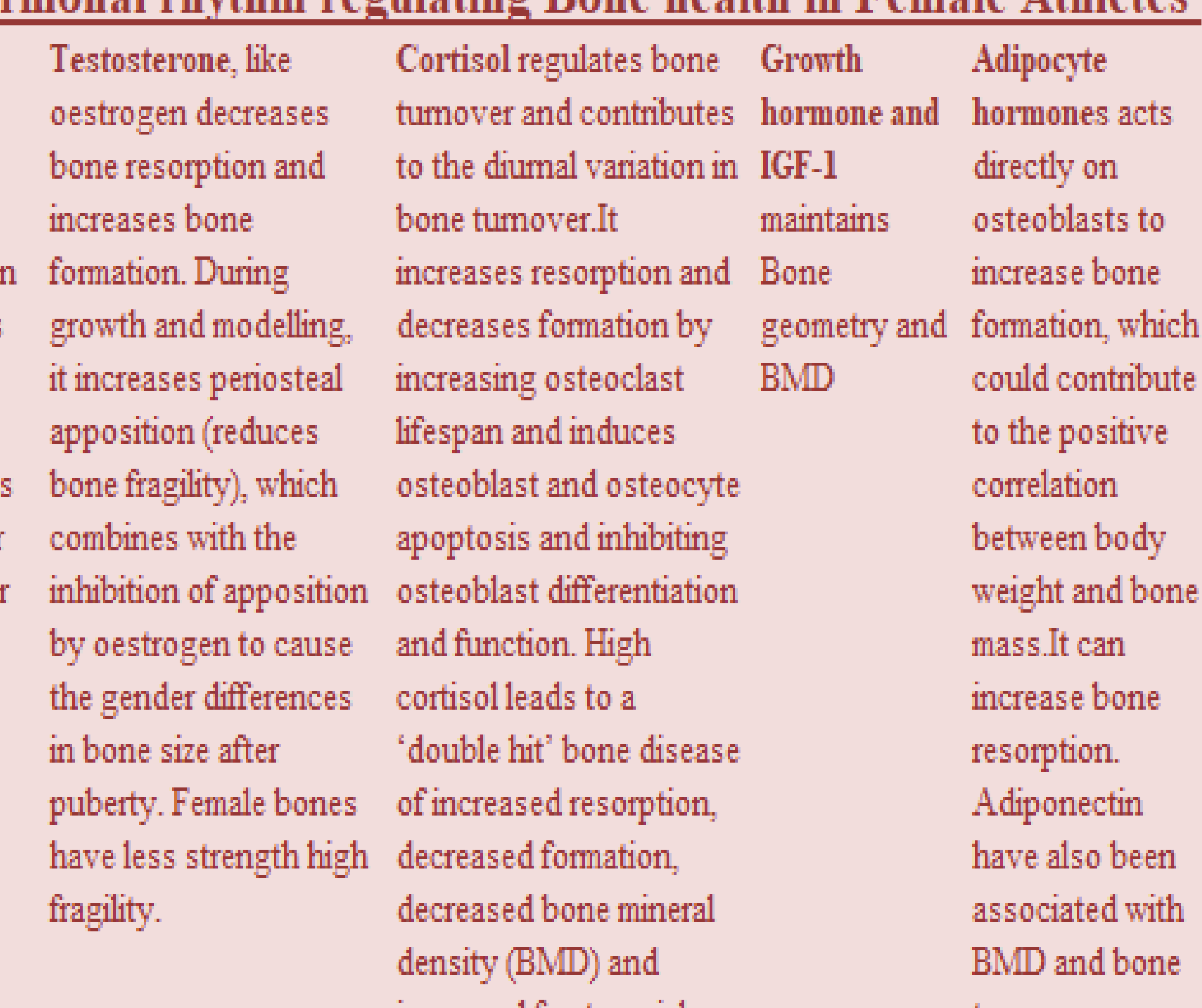

CONCLUSION

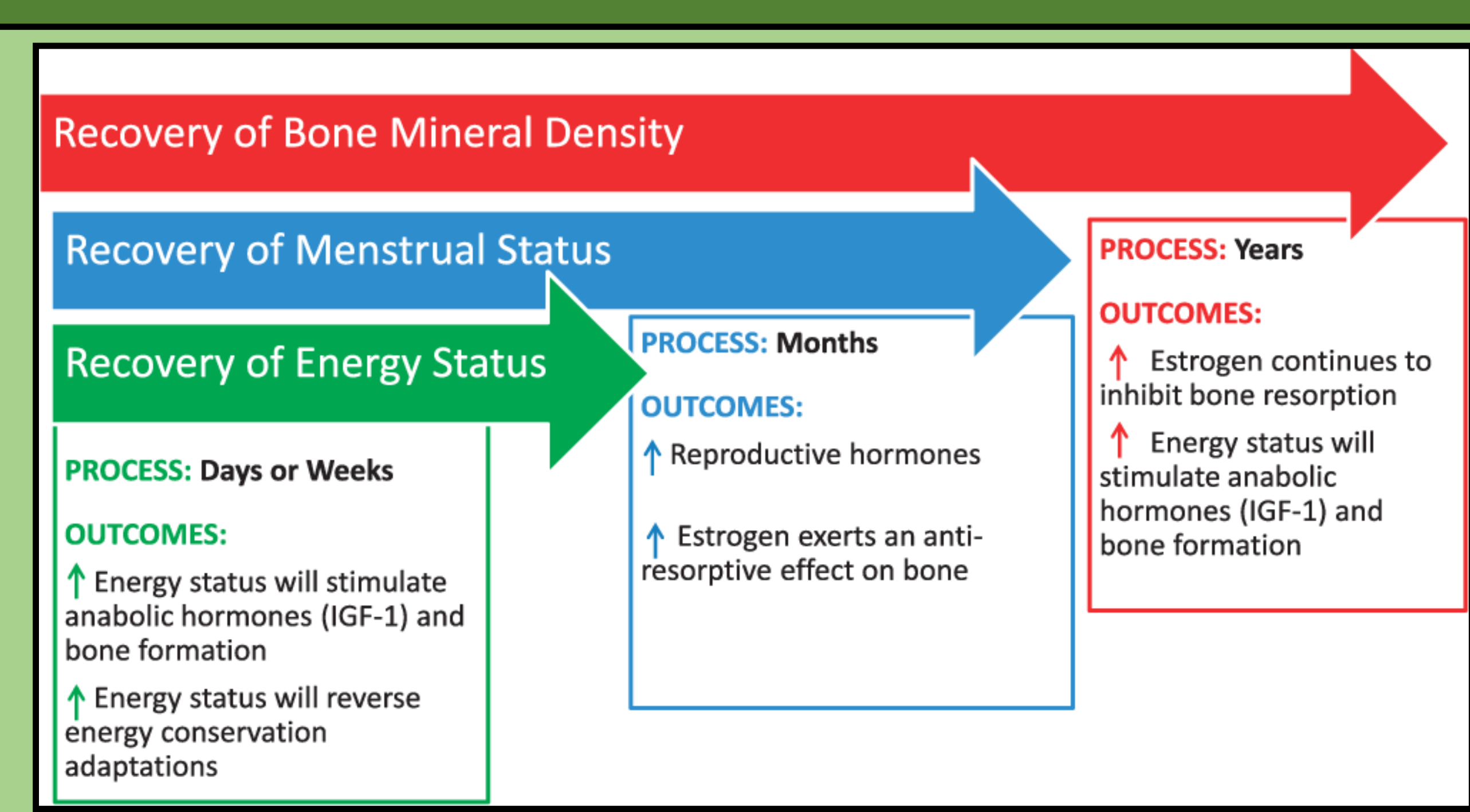

Female athletes are often dealing with low energy availability with or without eating disorders, functional hypothalamic amenorrhea, osteoporosis and random psychological problems alone or in combination to the other Triad components. An early intervention is disorders and pose significant health hazards. Regularly menstruating female athletes do
ditions not need to amend for menstrual cycle to exploit performance instead they can receive multi-disciplinary professional help to improve their performance and recover from the multi-cisciad.
Triad

\section{ACKNOWLEDGEMIENT}

I am sincerely thankful to my Professor, Dr. Asok Kumar Ghosh, for educating me with such an interesting topic of Female Athlete Triad and Puja Ghosh for helping me with the poster. Lastly, I am grateful to my family, friends and all my professors at RKMVERI for believing in me.

\section{REFERENCES:}

(3)

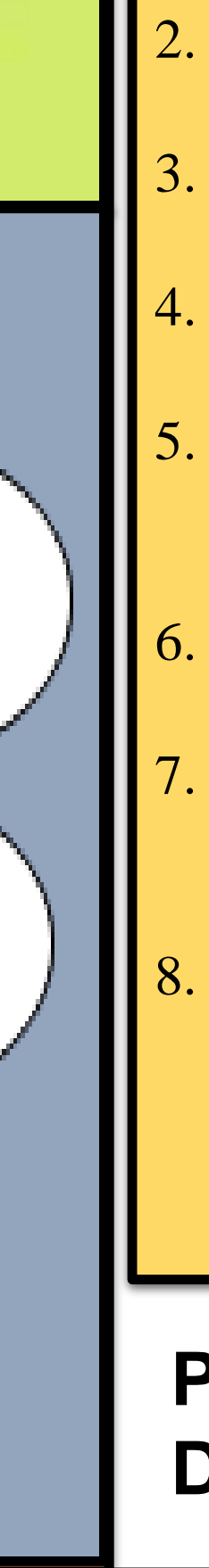

